\title{
Retos y desafíos de los posgrados en las universidades públicas del Siglo XXI
}

Leticia Salomón*, Jorge Amador**

\section{INTRODUCCIÓN}

En las últimas décadas se ha visto un desplazamiento acelerado de la importancia de los grados académicos requeridos por nuestras sociedades. El papel central que jugaron en su momento las titulaciones de nivel medio, relacionadas con los maestros, peritos mercantiles, bachilleres en ciencias y letras, secretarias y otros similares, fue superado rápidamente por las titulaciones universitarias a nivel de licenciatura, que llegaron a ocupar un lugar importante en materia de realización profesional y prestigio social durante muchos años. El último cuarto del siglo XX mostró en nuestra región el auge de los posgrados a nivel de especialidades y maestrías, lo que vino a desplazar la importancia social y académica de las licenciaturas que pasaron a ser un peldaño más en la carrera educativa, similar al papel que jugaron en su momento los bachilleratos de nivel medio, ubicados como el paso necesario para ingresar a la universidad. En el inicio del siglo XXI se produce una especie de boom de los posgrados, el cual comienza a desarrollarse en diversas áreas del conocimiento, en unas más que en otras, y comienzan a ocupar un lugar importante los doctorados y, en sociedades más desarrolladas, los pos- doctorados. Los posgrados que aparecen con tanta fuerza en nuestras universidades comienzan a ser impulsados por profesionales visionarios, académicos comprometidos y autoridades consecuentes con la época e inician sus actividades casi en solitario, sin mayores regulaciones, sin inserción en la estructura académica y al margen de las facultades, las cuales eran vistas con recelo por no haber dado el salto a grados académicos superiores a la licenciatura.

De esta manera van surgiendo nuestros posgrados en una especie de enclaves académicos, muy dinámicos hacia adentro de ellos y hacia otras universidades y países, pero muy cerrados hacia el resto de sus propias universidades, acentuando con ello la distancia entre el grado y el posgrado, rompiendo muchas veces, el continuum formativo de las áreas del conocimiento y desconociendo los procedimientos administrativos de las universidades, situación que caracterizó en mayor medida a los posgrados de universidades públicas. Los posgrados surgieron y se desarrollaron con el sello de la auto sostenibilidad que incluía no sólo el aspecto financiero sino también el capital humano que comenzó a darle forma al concepto de "profesor de posgrado", algunas veces proveniente de fuera de las universidades ("profesor visitante") y otras de la propia universidad, a quienes se contrataba específicamente para impartir una asignatura por la cual recibía un pago adicional dado que era una actividad al margen de su carga académica. Esta práctica se fue generalizando y dio lugar a profesores de posgrado sin arraigo en el mismo porque su paso por él era fugaz y, por

\footnotetext{
Universidad Nacional Autónoma de Honduras. Directora de Investigación Científica y Posgrados de la Universidad Nacional Autónoma de Honduras. investigacionunah.directora.Is@gmail.com

"Universidad Nacional Autónoma de Honduras. Profesor de la Dirección de Investigación Científica de la Universidad Nacional Autónoma de Honduras.. investigacionunah.gestion.ja@gmail.com
} 
ello, sin que pudiera darle al mismo algo más que sus conocimientos especializados. Este hecho, repetido una y otra vez, fue afectando la calidad académica de los posgrados, los cuales vieron reducida su actividad investigativa y las demás asociadas a la misma, como encuentros académicos, publicaciones científicas de calidad, grupos de investigación y asesorías de tesis, lo cual fue colocándolos en desventaja con respecto a posgrados similares de otras universidades y países. El auge de los posgrados fue seguido de una etapa de deterioro que iba paralelo a los procesos de evaluación y acreditación que comenzaron a jugar un papel central en el proceso de búsqueda de la calidad. La situación llegó a agudizarse tanto que se expresaba en el bajo nivel de los estudiantes que ingresaban pero, lo que era más preocupante, en el bajo nivel de los estudiantes que egresaban, produciéndose un desplazamiento hacia abajo en los indicadores de calidad, cuando los posgrados comenzaron a perder sus status académico. Todo ello, sumado a la masificación, a la profundidad de las brechas con las facultades y a dificultades propias de los planes de estudio, se tradujo en cantidades crecientes de estudiantes que finalizaban sus estudios pero no defendían sus tesis, dando lugar al fenómeno de la ineficiencia terminal que fue constituyéndose en un indicador negativo de la gestión de posgrados.

De forma paralela, el Estado, los sectores productivos, los sectores sociales y la cooperación internacional, comenzaron a demandar profesionales especializados con estudios de posgrado, de acuerdo a las nuevas necesidades del contexto socio-económico del país, lo que propició el aparecimiento de múltiples posgrados, en el ámbito público y privado, no siempre con la calidad requerida, y comenzaron a bajar los requisitos académicos de las tesis para propiciar la rápida obtención de los títulos respectivos. Sin asumirlo plenamente, se fue produciendo una diferenciación creciente entre posgrados académicos o científicos y posgrados profesionales o profesionalizantes $y$, de forma equivocada, se fueron diferenciando los posgrados denominados "teóricos", de los posgrados denominados "prácticos", afectando en el proceso la calidad de la investigación científica, por un lado, y la calidad de la formación profesional, por el otro. En la actualidad se plantea la necesidad de que las universidades asuman con mayor compromiso la apertura y desarrollo de los posgrados, complementado con el papel que juegan las instancias evaluadoras y el papel central de las instancias de gestión de los posgrados dentro de la estructura académica de las universidades.

Para ello se espera tener claro el posicionamiento institucional de una universidad ante los posgrados y elaborar una política que precise con claridad lo que se quiere hacer, cómo, cuándo, con qué recursos y en cuanto tiempo, todo ello expresado en una visión estratégica a cinco años para orientar la planificación, los recursos y la asignación de personal. La política y la visión estratégica deben complementarse con las líneas o temas de investigación en los cuales quiere hacer incidencia una universidad a través de sus posgrados, sean estos académicos o profesionalizantes y recolocar la atención en la investigación científica, la vinculación con el Estado, los sectores productivos y los sectores sociales, la normativa adecuada a través de reglamentos, manuales e instructivos, y sobre todo, el fortalecimiento de la instancias de gestión institucional de los posgrados, que son las llamadas a darle seguimiento a los mismos, evaluarlos, regularlos, apoyarlos e impulsarlos. 


\section{DOCENCIA, INVESTIGACIÓNY VINCULACIÓN EN LOS POSGRADOS DEL SIGLOXXI}

Los posgrados, concebidos como instancias superiores de la enseñanza universitaria, deben cumplir con las tres funciones básicas de la universidad, como son la docencia, la investigación científica y la vinculación universidad-sociedad, las cuales se expresan o deben expresarse de la siguiente manera:

\section{La docencia en posgrado}

La docencia en posgrado supone una calidad superior a la docencia en el grado y un abordaje superior en profundidad teórica y metodológica, que debe ser consecuente con el continuum académico entre grado y posgrado. Cuando se produce un aumento de la calidad en la docencia a nivel de grado y la reducción de la calidad a nivel de posgrado en disciplinas específicas, tiende a generalizarse la idea de que la enseñanza en el posgrado experimenta un desplazamiento cualitativo hacia abajo el cual repercute negativamente en la calidad de los posgrados. Un gran paso adelante significó la exigencia de que los profesores que se dedicaban a la docencia debían tener un grado similar o superior a la carrera en donde la impartían. En el caso de los posgrados esta disposición significó un salto cualitativo que repercutió positivamente en el desarrollo académico de los mismos; sin embargo, la exigencia no incluyó que los profesores tuvieran la condición de investigadores con líneas de investigación claramente delimitadas y en estrecha relación con el tema del posgrado, lo que se tradujo en una mejora sustancial de la docencia que no repercutió en una mejora sustancial de la investigación científica. La participación de profesores procedentes de otras universidades y otros países constituyó un impulso más en pos de la calidad. Muchos posgrados se iniciaron y se mantuvieron durante mucho tiempo con profesores amigos de instituciones y países amigos, con lo cual se iniciaba y fortalecía la condición internacional que posteriormente se convertiría en un indicador de calidad de los posgrados. El aspecto central a considerar, situación que en algunos casos no se produjo, fue la correcta articulación entre profesores nacionales e internacionales, tratando de descubrir, atraer y capacitar a especialistas nacionales que vinieran a complementar y contextualizar la enseñanza de los profesores internacionales.

Este proceso, más evidente en unos posgrados que en otros, vino a promover cierta vulnerabilidad hacia el exterior y la urgencia de conseguir sustitutos de última hora, sin una visión planificada que permitiera una búsqueda más cuidadosa. El desarrollo de las comunicaciones y el fortalecimiento de las tecnologías correspondientes introdujo un elemento de innovación en la modalidad de la docencia en los posgrados, permitiendo que un profesor de una universidad en un punto del planeta, el continente o la región, pudiera impartir conferencias, seminarios, cursos o asesorías utilizando la virtualidad como un recurso total 0 parcial dándole sustento a la bimodalidad e incorporando como requisitos de la docencia en posgrado nuevas habilidades y manejos tecnológicos.

\section{La investigación científica}

En el contexto anterior, el desarrollo de la investigación científica como una de las tres 
funciones básicas de la universidad ha experimentado un desarrollo diferenciado, en unos casos, muy fuerte y, en otros casos, muy débil. Por lo general, la investigación se ha reducido a las tesis que los estudiantes desarrollan como requisito de graduación y muy poco o nada se concentra en actividades de grupos de investigación que desarrollan proyectos, escriben artículos científicos, dictan conferencias sobre sus temas de investigación, organizan congresos o simposios y publican en revistas indexadas y arbitradas. Los trabajos que se realizan como parte de la responsabilidad estudiantil en las respectivas asignaturas se pierden en los requisitos de evaluación y no logran convertirse en artículos publicables con la asesoría de los respectivos profesores, minimizando de esa manera el recurso de la investigación educativa como uno de los pilares de la investigación en los posgrados. Lo mismo ocurre con la inexistencia de revistas propias con la calidad necesaria que recojan los mejores artículos de los profesores nacionales e internacionales y de los estudiantes y se conviertan en puntos de debate y en referentes institucionales sobre los temas especializados en que se manejan.

Otro aspecto central de esta debilidad es la ausencia de grupos de investigación en los posgrados que incluyan profesores/investigadores y estudiantes que desarrollen sus tesis dentro de las líneas de investigación de los grupos. Publicar se convierte en una actividad inherente a los posgrados y tiene un doble propósito: Contribuir al conocimiento de los problemas nacionales (lo que exige publicar adentro del país) y contribuir al desarrollo científico-tecnológico (lo que exige publicar afuera del país). Ambos propósitos devienen obligaciones para los profesores/investigadores de los posgrados, dada la obligación de atender las necesidades del país desde la academia y atender con similar empeño los desafíos del desarrollo científico-tecnológico. Un desafío adicional se relaciona con la creciente necesidad de hacer investigación interdisciplinaria, interuniversitaria e internacional. Los esfuerzos colaborativos deben partir de las diferentes disciplinas dentro de un área del conocimiento y continuar con la conjunción de otras disciplinas que exigen abordajes integrales de los temas específicos. El siguiente paso es buscar pares temáticos en otras universidades del país o del mundo y realizar esfuerzos colaborativos que combinen situaciones geográficas, económicas y sociales diferentes que posibiliten estudios comparativos con enfoques teóricos y abordajes metodológicos diferenciados. El establecimiento de prioridades de investigación en la universidad, a nivel general, y en los posgrados a nivel particular, permite establecer áreas de incidencia que incluyan a investigadores con sus proyectos y estudiantes con sus temas de tesis u otros trabajos científico-educativos.

\section{La vinculación universidad-sociedad}

La universidad cuenta con una tercera función que también ha permanecido marginada a nivel de posgrados. Se trata de la vinculación de la universidad con el Estado, los sectores productivos y los sectores sociales, lo cual tiene múltiples maneras de asumirse en los posgrados. Para empezar, cada uno de esos sectores tiene intereses específicos en el producto que sale de la universidad, sean profesionales (capital humano) artículos, libros 0 invenciones (capital intelectual) mismos que pueden ser objeto de apoyos, convenios 0 alianzas, sea para impulsar especialidades, maestrías o doctorados, o para financiar 
investigaciones en temas coincidentes de la agenda nacional y la agenda universitaria.

La definición de prioridades nacionales en ciencia y tecnología, y el establecimiento de zonas prioritarias del desarrollo nacional por parte del Estado, constituye un punto de partida para la articulación de esfuerzos en virtud de las coincidencias de intereses y las capacidades acumuladas. La universidad posee recursos en docencia que pueden utilizarse para formar 0 capacitar; posee investigadores que pueden desarrollar proyectos propios, asesorar y evaluar investigaciones realizadas y preparar una oferta de investigaciones por realizar para atender alguna necesidad específica; igualmente cuenta con los estudiantes de posgrado que pueden realizar prácticas profesionales en instituciones del Estado, sectores productivos y sectores sociales, e insertarse en proyectos de desarrollo a nivel local, regional o nacional. Un mundo de posibilidades se abre para los posgrados en lo que parece ser una oportunidad inexplorada de acercamiento y trabajo colaborativo estructurado alrededor de la función de vinculación. La apertura de la universidad al país y al mundo conlleva áreas de trabajo que pueden resultar muy positivas siempre y cuando se tenga presente el concepto de universidad con visión de mundo y desarrollo científico y tecnológico.

\section{Grado de Exigencia en Formación e Investigación en los Posgrados}

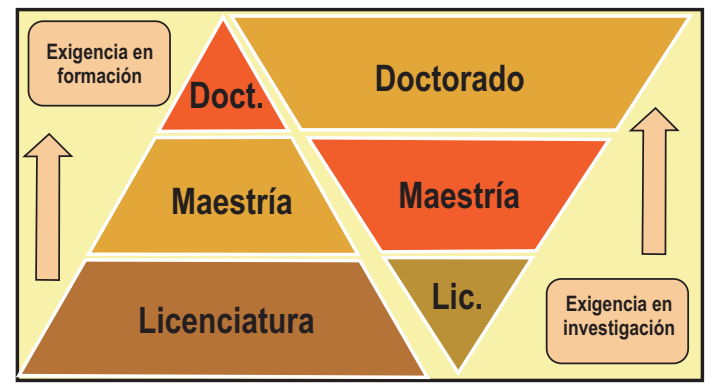

\section{La visión integral}

Al hablar de funciones universitarias y de los puentes que unen los grados con los posgrados, se vuelve una obligación buscar puntos de encuentro 0 , por lo menos, los puntos de intersección que faciliten el desarrollo de una concepción integral para desarrollar los posgrados y obtener un mayor impacto académico. Para empezar, debemos estar conscientes que los requerimientos en formación e investigación son y deben ser diferenciados de acuerdo al grado académico que se esté considerando. Por ejemplo, vemos que el componente de formación es grande en la licenciatura, disminuye un poco en la maestría y disminuye mucho más en el doctorado, a diferencia de lo que ocurre con el componente de investigación que es reducido en el primer nivel y se va ampliando conforme asciende en la escala. Esto nos está indicando que a nivel de licenciatura, que es el nivel anterior que debe cubrirse antes de pasar a la maestría, deben consolidarse conocimientos metodológicos básicos que permitan a los estudiantes del nivel realizar de manera exitosa una primera aproximación a la investigación en el ámbito específico de los requisitos curriculares. 
Las debilidades de este nivel en materia de investigación repercutirán en la maestría y obligarán a los profesores a disminuir el nivel de rigurosidad metodológica por el riesgo de quedarse sin estudiantes. Por otro lado, la existencia de instancias académicas que gestionan las funciones básicas de la universidad como compartimentos estancos sin comunicación entre sí, impide que la universidad se proyecte con más intensidad a la vida nacional e internacional y que pueda impulsar actividades y proyectos integrales en los cuales se desdibuja la línea que separa una de la otra. Lo anterior indica que la misma razón de la interdisciplinariedad, los grupos de investigación y el trabajo colaborativo que exigimos a los profesores, investigadores y estudiantes, debe aplicarse a las instancias de gestión académica que se encargan de impulsar las tres funciones básicas de la universidad, y con más intensidad en los posgrados.

\section{LA GESTIÓN INSTITUCIONAL DE LOS POSGRADOS EN EL SIGLOXXI}

\section{La gestión organizativa}

El grado de desarrollo de los posgrados es aún un tema pendiente en algunas universidades latinoamericanas, pues pese a la existencia de diferentes escenarios con distintos grados de avance, no parece existir una clara percepción de los mismos desde una perspectiva integral, coherente, armónica y estrechamente vinculada a las tres funciones básicas de la universidad. El primer punto es el establecimiento de una política de posgrados en cada universidad para precisar lo que se quiere hacer en un horizonte mínimo de cinco años, cómo hacerlo, para qué, en cuanto tiempo y con qué recursos, estableciendo los ejes sobre los cuales deben articularse, los aspectos que requirieran fortalecerse y los errores que deban enmendarse. El nacimiento de un posgrado fuera de la estructura académica universitaria trae consigo desventajas más que ventajas y se expresa en siete debilidades que impactan negativamente sobre el desarrollo eficiente de los mismos: a) posgrados desarraigados de la facultad, b) posgrados sin el continuum disciplinar (grado-posgrado), c) posgrados sin profesores locales que permitan la contextualización del conocimiento, d) posgrados sin vínculos con los investigadores de la facultad, e) posgrados sin vida académica, que no desarrollan eventos de investigación, divulgación, publicación científica, etc., f) posgrados que no se rediseñan y buscan la acreditación, g) posgrados con coordinadores que no son líderes en sus facultades, 0 en algunos casos no tienen la trayectoria académica para conducir una carrera de este tipo.

En algunos casos se llega al extremo de concentrar los programas de posgrado en una facultad de posgrado, con lo que se profundiza la distancia y la comunicación entre grado y posgrado, y se da el caso de un decanato sin profesores propios y un desarrollo de posgrados sin una mirada integral del desarrollo disciplinar de las ciencias. Uno u otro modelo no es aceptable teórica, filosófica ni administrativamente; las carreras de posgrado, como las carreras de grado, deben permanecer y ser conducidas en una facultad que como tal desarrolla áreas del conocimiento, asumiendo la existencia de una entidad normativa dentro de la universidad (a nivel de Vicerrectoría o Dirección) que regule su quehacer y vele por su correcto desempeño. 


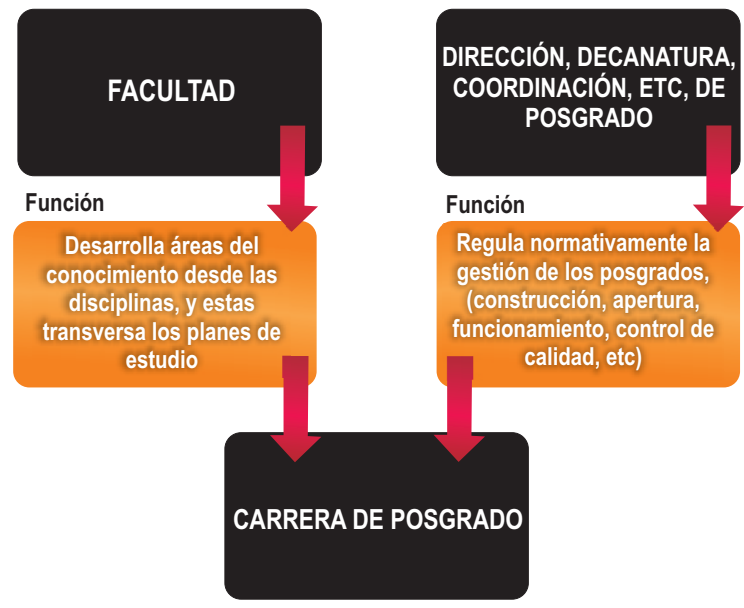

\section{La gestión financiera}

En Centroamérica se presenta un problema particular con los programas de posgrado: la mayoría nace desvinculada de las facultades por la idea de auto sostenibilidad asociada a los mismos, en una especie de autonomía que pretende, y muchas veces consigue, la autonomía académica y financiera para su desarrollo. Este proceso tiende a desconocer la normativa que regula sus procedimientos, la cual, en el caso de las universidades públicas, es la misma que regula al Estado. Buena parte de la explicación se encuentra en la nebulosa que envuelve una actividad privada (pago por un servicio) en el seno de un espacio público, mismo que llega a percibirse como un contrato de arrendamiento que se traduce en el pago de un porcentaje de lo recaudado a cambio del uso de las instalaciones y servicios de la universidad, sin terminar de percibir la dimensión de la cobertura institucional de su funcionamiento. La situación se complica más y su inserción institucional se desdibuja mucho más cuando dichos programas surgen vinculados a personas, instituciones $u$ organismos que gestionan 0 aportan al financiamiento de tales carreras, creando un sentido de propiedad que tiende a profundizar la autonomía y el desconocimiento de procedimientos de regulación o inserción institucional.

Lo anterior se agrava más si consideramos que la inversión del Estado en educación superior se focaliza en las carreras de pregrado y grado, obligando a los posgrados a autofinanciarse, sin hacer la diferenciación entre aquellos posgrados de fácil recuperación de lo invertido y los que no lo son, y entre aquellos necesarios para el desarrollo de la ciencia, la tecnología y las humanidades, y de la sociedad en su sentido integral, de aquéllos que responden a necesidades específicas de los sectores productivos. En consecuencia, las universidades públicas devienen obligadas a diferenciar las situaciones planteadas y potenciar el financiamiento de los posgrados organizados en cuatro categorías: 1) los posgrados sensitivos para el desarrollo de la ciencia y la tecnología, incluyendo las ciencias humanas y sociales que financieramente no son rentables para los estudiantes por la lenta recuperación de la inversión: químicos, físicos, matemáticos, filósofos, literatos, historiadores y otros 2) los posgrados que forman los profesionales requeridos por el Estado, promoviendo su 
fortalecimiento y desarrollo: ingenieros, médicos, economistas, enfermeras, pedagogos y otros; 3) los posgrados que fortalecen el pensamiento crítico y llegan a constituir los tanques de pensamiento que desarrollan teoría aplicada a los procesos económicos, políticos y sociales, y que generan propuestas, cuestionamientos y planteamientos a los tomadores de decisiones; y c) los posgrados demandados por el sector productivo cuyos graduados se insertan rápidamente en el campo laboral.

El establecimiento de alianzas estratégicas facilitaría de gran manera el financiamiento de los programas de posgrado en sus diferentes categorías: las universidades estatales deben apostar a financiar programas requeridos por la ciencia, la tecnología y las humanidades (primer grupo) y los que desarrollan el pensamiento crítico (tercer grupo) mientras que el Estado podría co-financiar el segundo grupo y el sector productivo podría hacer lo mismo con el cuarto grupo, teniendo en cuenta que los estudiantes siempre deberán pagar una parte del valor del programa de posgrado cuyo monto dependerá de la pertinencia del mismo y de las alianzas establecidas.

\section{La gestión curricular}

Los planes de estudio de las carreras de posgrado deben responder a la política curricular de las universidades, todas ellas alineadas con los modelos pedagógicos actuales que responden al enfoque constructivista humanista, en el que sus pilares, según Delors (1994), son el de "aprender a ser", "aprender a conocer", "aprender a hacer" y "aprender a vivir juntos": estos deberían de ser los pilares de la construcción curricular en los planes de estudio. No debe considerarse la gestión curricular como el simple cumplimiento de requisitos administrativos en el que los procesos y las formas deben ejecutarse como cada entidad lo estipula, sino una gestión curricular de calidad que se fundamente en: 1) la política curricular universitaria concretada en el modelo educativo propio de cada institución; 2) la estructura académica que desarrolla el currículo en cada institución; 3) la formación y capacitación en currículo; 4) el fortalecimiento de las prácticas pedagógicas universitarias; 5) la capacidad de diseñar y rediseñar planes de estudio de acuerdo a las exigencias estatales, sociales, productivas y académicas.

La política curricular universitaria es la definición pedagógica que define cada uno de los elementos curriculares, es decir, se considera la filosofía de la educación que cada universidad se apropia y busca estrategias para su concreción; esto se relaciona con el ideal de profesional que se pretende formar y se identifica con un enfoque pedagógico y los diferentes diseños curriculares que concretan dicho enfoque. De acuerdo a ella, se debe especificar quién o quiénes, según la estructura académica universitaria, son los llamados a la construcción y desarrollo curricular, y cuáles serán las disciplinas que se desarrollarán a través de los planes de estudio para la formación de profesionales; además, cada área del conocimiento organizará y fortalecerá estructuras para el diseño y desarrollo curricular. Las universidades deben aspirar a capacitar pedagógicamente a los docentes que integran la estructura de diseño y desarrollo curricular, recordando que ellos son especialistas en sus áreas, no en currículo, y, por lo tanto, se deben proporcionar los elementos técnicos que ellos deberán aplicar en sus respectivas áreas del conocimiento. En muchos casos, las 
universidades se plantean un modelo educativo, pero no especifican la forma en que el currículo debe entrar al aula, es decir, la forma en que éste se concreta, lo que se relaciona directamente con el fortalecimiento de las prácticas pedagógicas universitarias, como parte fundamental de una reforma educativa, para evitar la distancia que separa el enfoque que se enuncia (constructivista, p.e.) y lo que se aplica en el aula (examen escrito, p.e), aspecto que, por lo general, contradice el enfoque enunciado. Lo anterior nos lleva a plantear que las prácticas pedagógicas universitarias que deben fortalecerse al momento de apropiarse de un modelo educativo, que también aplica a los posgrados, deben ser las siguientes: la planificación docente, la metodología, los recursos, la evaluación y el rol del docente y estudiante.

La construcción de un plan de estudios debe iniciar con la conceptualización del profesional requerido y su campo de desarrollo como tal, para considerar su quehacer y delimitar la construcción curricular. En el caso de los posgrados, la clasificación de académicos 0 profesionalizantes le da un giro importante a la determinación del diseño curricular, la selección de contenidos, las formas de salida, la intensidad de la investigación científica y las actividades de vinculación con la sociedad.

\begin{tabular}{|c|c|c|}
\hline Concepto & Posgrados Académicos & $\begin{array}{l}\text { Posgrados } \\
\text { Profesionalizantes }\end{array}$ \\
\hline $\begin{array}{l}\text { Determinación de } \\
\text { espacios curriculares }\end{array}$ & Teóricos - Prácticos & Prácticos - Analíticos \\
\hline Contenidos & $\begin{array}{l}\text { Fundamentos de las } \\
\text { disciplinas para la } \\
\text { realización de investigación } \\
\text { científica. }\end{array}$ & $\begin{array}{l}\text { Fundamentos de las } \\
\text { disciplinas para la } \\
\text { intervención en escenarios } \\
\text { de la realidad. }\end{array}$ \\
\hline Evaluación & $\begin{array}{l}\text { Basada en fundamentos } \\
\text { teóricos que debe dominar el } \\
\text { estudiante para el desarrollo } \\
\text { de las ciencias y el abordaje } \\
\text { de la realidad nacional } \\
\text { desde su perspectiva } \\
\text { profesional. }\end{array}$ & $\begin{array}{l}\text { Basado en capacidades del } \\
\text { estudiante para resolver } \\
\text { problemas cotidianos del } \\
\text { quehacer profesional, } \\
\text { aplicando los conocimientos } \\
\text { adquiridos }\end{array}$ \\
\hline Investigación Científica & Fundamentalmente básica & Fundamentalmente aplicada \\
\hline Formas de salida & $\begin{array}{l}\text { Evidencias de producción } \\
\text { científica con rigurosidad } \\
\text { metodológica }\end{array}$ & $\begin{array}{l}\text { Evidencias de aplicación } \\
\text { metodológica para la } \\
\text { resolución de problemas }\end{array}$ \\
\hline
\end{tabular}

Las universidades deben considerar estrategias que promuevan el rediseño de los planes de estudio de manera ágil y eficaz, y procurar que en periodos cortos de tiempo puedan aplicarse dichas reformas, sin olvidar que en esta era globalizada los contenidos y las competencias profesionales exigidas son rápidamente cambiantes. Dadas las condiciones de un mundo en constante transformación y la velocidad con que se produce conocimiento nuevo, una universidad con planes de estudio de posgrado estáticos por más de cinco años 
corre el riesgo de que el programa pierda la pertinencia de la formación profesional y quede desactualizado, lo que obliga a la revisión periódica, la innovación constante y la supervisión eficiente, cuidando que los procesos duren un tiempo suficientemente corto para que no se traslape con nuevas exigencias de actualización curricular.

\section{La Internacionalización en la gestión}

La dimensión internacional debe ser una parte intrínseca, junto con la calidad y la pertinencia, de las instituciones y de los sistemas de educación superior (CMES, París, 1998). Debe considerarse la internacionalización como la oportunidad para reducir las brechas entre los países desarrollados y los que están en vía de desarrollo; la conceptualización de una visión internacional en los planes de estudio, unida a la movilidad de doble vía de estudiantes, profesores e investigadores que, a su vez, asesoran a estudiantes y comparten experiencias, enfoques teóricos y abordajes metodológicos con sus pares internacionales, son un indicador de la internacionalización de la gestión de los programas de posgrado.

La internacionalización va más allá de la movilidad. Es conectar la universidad con el mundo e incorporar visiones de otras realidades para conocer y comparar cómo avanzan procesos, y se desarrollan enfoques y abordajes en situaciones similares, recordando que cuando se internacionaliza uno de los actores, más tarde o más temprano se internacionaliza también el currículo o se fortalece su grado de internacionalización. Este proceso se ve estimulado y potenciado por las facilidades que nos brindan las comunicaciones, los convenios con otras universidades y el uso adecuado de las tecnologías de la información para propiciar la impartición de cursos, conferencias y asesorías, las publicaciones conjuntas y la conformación de grupos de investigación internacionales para proyectos específicos en torno a líneas de investigación que repercutan en beneficio para los posgrados de uno u otros países.

Un punto central a definir es el balance existente entre lo nacional y lo internacional, y cómo debe irse consolidando el componente nacional para aprovechar de mejor manera el componente internacional. En otras palabras, un posgrado debe demostrar inicialmente su grado de internacionalización con el peso de los profesores, investigadores y estudiantes que vienen de otros países a potenciar la calidad de la docencia, la investigación científica de profesores y estudiantes, y la visibilidad de su vinculación con sectores estratégicos del Estado, sectores productivos y sectores sociales. En la medida en que se fortalece el posgrado, la internacionalización debe complementarse con la movilidad de profesores, investigadores y estudiantes nacionales hacia otras universidades y países que comienzan a reconocer en ellos su calidad docente e investigativa, en el caso de los primeros, y la calidad de los planes de estudio, en el caso de los últimos. Comienza a percibirse con mucha fuerza esto último como un indicador de calidad de un posgrado, lo que abre posibilidades de atracción de profesores e investigadores cuyas universidades estimulan y reconocen la movilidad hacia afuera. 


\section{El aseguramiento de la calidad}

En los centros de educación superior de América Latina y el Caribe se impulsan los procesos de evaluación de la calidad de la educación del nivel debido a la preocupación que surge con la crisis económica que caracterizó los años setenta y ochenta, y con la sustitución del concepto de Estado benefactor por el de Estado evaluador, en materia de educación, en el que las restricciones de financiamiento público que sufre la educación superior fue asociada a percepciones sobre su baja calidad y falta de pertinencia.

Todo proceso evaluativo pretende evidenciar el grado de calidad asociado, en este caso, de la educación superior. De la Garza/Tünnermann nos explica que cuatro criterios son los determinantes para la evaluación de la calidad, siendo éstos la relevancia, eficacia, eficiencia y equidad, los cuales se pueden aplicar a los tres momentos que sirven para asegurar la calidad de un posgrado: la autoevaluación, la evaluación y la acreditación. La autoevaluación incluye indicadores internos que aseguran el funcionamiento eficiente de un programa de posgrado, y se constituyen en el insumo de un plan de mejoramiento, que desde una mirada interna sirva para potenciar el programa, elevando la calidad en el servicio. La evaluación, la cual es externa al programa, aplicada de forma institucional, por la entidad rectora de los posgrados en la universidad, o bien por la instancia acreditadora nacional, cualquiera de las cuales, además de evaluar y asegurar la eficacia del programa, podrá determinar la permanencia del mismo dentro de la oferta, teniendo como indicadores principales, la gestión financiera, la organizativa, el porcentaje de graduación y la visibilidad del programa, este último a través de la publicación de resultados de investigación de profesores y estudiantes, la coordinación o participación en eventos de divulgación científica dirigidos a los potenciales beneficiarios ya sean del sector estatal, social o productivo, y el reconocimiento social y estatal del posgrado como un referente académico en el área de su especialización.

La acreditación, como lo estipula De la Garza/Tünnermann, tiene tres tipos de sistemas: a) los organismos gubernamentales, b) los organismos intermedios y c) los organismos profesionales/académicos, quienes "certifican externamente" la calidad de los programas de posgrados, controlándola y garantizándola. Tiene como base fundamental los dos niveles anteriores a él (autoevaluación y la evaluación), incluyendo en este último nivel la evaluación porpares.

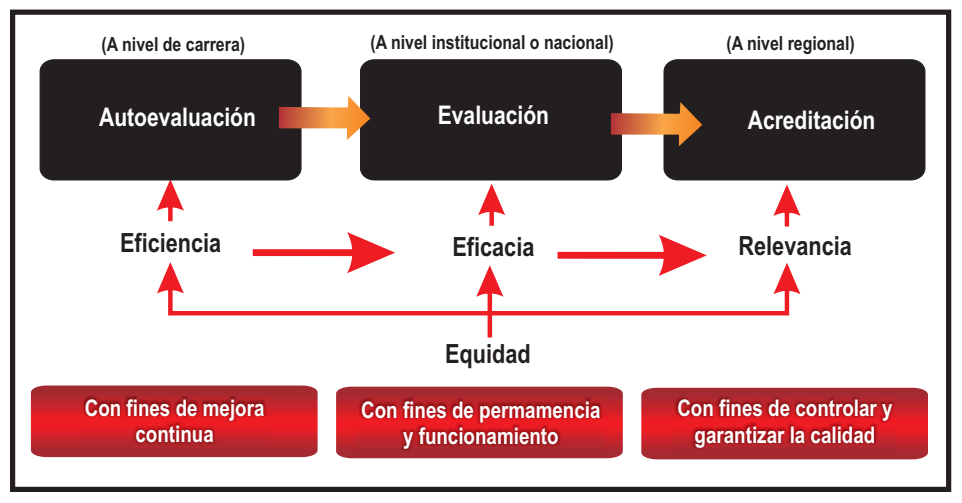




\section{RETOSYDESAFÍOS}

Las carreras de posgrado merecen una mayor atención dentro de las universidades estatales. Para que ello suceda se deben considerar los siguientes retos y desafíos:

- Fortalecer la estructura académica universitaria, insertando en ella los programas de posgrado según las disciplinas que estos desarrollan, cuyas bases se encuentran en las carreras de pregrado y grado.

- Definir una política de posgrados que contemple el posicionamiento institucional y su desarrollo en una visión no menor a cinco años.

- Organizar los posgrados en las cuatro categorías enunciadas para la búsqueda de financiamiento, superar el concepto de que todos los posgrados son auto sostenibles, y asumir que todos los posgrados van ligados a necesidades concretas del Estado, los sectores productivos, los sectores sociales y la ciencia.

- Definir una política curricular de cada universidad y, después de ello, preparar los instrumentos necesarios para concretarla y hacer que las reformas educativas universitarias entren al aula.

- Desarrollar el concepto de internacionalización y aplicarlo a todos los programas de posgrado para que estos se potencien a través de ella.

Asegurar el acompañamiento técnico en los procesos de autoevaluación, evaluación institucional y el de acreditación para la certificación de los programas de posgrado, procurando mantener estándares de calidad en los mismos.

\section{BIBLIOGRAFÍA}

Consejo Nacional de Ciencia y Tecnología. (2012). Marco de Referencia para la evaluación y seguimiento de programas de posgrado en las modalidades a distancia y mixta. México: CONACYT.

lesalc - UNESCO. (2008). La Educación Superior en América Latina y el Caribe: diez años después de la Conferencia Mundial de 1998. (C. Tünnermann Bernheim, Ed.).

OEI. (2012). Ciencia, Tecnología e Innovación para el desarrollo y la cohesión social . Madrid: OEl.

UNESCO - EDUCACIÓN. (2004). Educación Superior en una sociedad mundializada. Paris: UNESCO.

UNESCO. (2009). Conferencia Mundial sobre la Educación Superior 2009: La nueva dinámica de la educación superior y la investigación para el cambio social y el desarrollo. Paris: UNESCO. 